\title{
Experimental Study on Localized Surface Plasmon Mode Hybridization in the Near and Mid Infrared
}

Johannes Srajer, ${ }^{\text {a,b }}$ Andreas Schwaighofer, ${ }^{\mathrm{a}}$ Georg Ramer, ${ }^{\mathrm{c}}$ Pinar Frank, ${ }^{\mathrm{a}}$ Bernhard Lendl, ${ }^{\mathrm{c}}$ Christoph Nowak, *a,b

${ }^{a}$ Austrian Institute of Technology GmbH, AIT, Donau-City Str. 1, 1220 Vienna, Austria.

${ }^{\mathrm{b}}$ Center of Electrochemical Surface Technology, CEST, Viktor-Kaplan-Straße 2, 2700

Wiener Neustadt, Austria

${ }^{\mathrm{c}}$ Institute of Chemical Technologies and Analytics, Vienna University of Technology, Getreidemarkt 9/164 AC, 1060 Vienna, Austria

Johannes Srajer and Andreas Schwaighofer equally contributed to the work.

This is a post-peer-review, pre-copyedit version of an article published in Plasmonics. The final authenticated version is available online at: DOI: 10.1007/s11468-014-9690-7. 


\begin{abstract}
We investigate plasmon excitations within a regular grating of double layered gold/insulator nanoparticles in the infrared and visible spectral region. Provided a flat gold film as substrate, strong coupling between the localized surface plasmon modes and their image like excitations in the metal is observed. The interaction results in a strong red shift of the plasmon mode as well as the splitting of the modes into levels of different angular momenta, often referred to as plasmon hybridization. The diameters of the nanoparticles are designed in a way that the splitting of the resonances occurs in the spectral region between 0.1 to $1 \mathrm{eV}$, thus being accessible using an infrared microscope. Moreover, we investigated the infrared absorption signal of gratings that contain two differently sized nanoparticles. The interaction between two autonomous localized surface plasmon excitations is investigated by analyzing their crossing behavior. In contrast to the interaction between localized surface plasmons and propagating plasmon excitations which results in pronounced anticrossing, the presented structures show no interaction between two autonomous localized surface plasmons. Finally, plasmon excitations of the nanostructured surfaces in the visible spectral region are demonstrated through photographs acquired at three different illumination angles. The change in color of the gratings demonstrates the complex interaction between propagating and localized surface plasmon modes.
\end{abstract}

\title{
Keywords
}

Surface enhancement, plasmon excitation, localized surface plasmon, plasmon hybridization, IR spectroscopy

$\begin{array}{ll}\text { Abbreviations } & \\ \text { IR } & \text { Infrared } \\ \text { VIS } & \text { Visible } \\ \text { FWHM } & \text { Full width at half maximum } \\ \text { FDTD } & \text { Finite difference time domain } \\ \text { FPA } & \text { Focal plane array } \\ \text { MCT } & \text { Mercury cadmium telluride } \\ \text { LSP } & \text { Localized surface plasmon } \\ \text { PSP } & \text { Propagating surface plasmon }\end{array}$




\section{Introduction}

What started with changing the color of glass thousands of years ago, led to a revolution in biological and chemical sensing over the past view decades $[\underline{1}, \underline{2}]$. The strong interaction between plasmons and surrounding molecules enabled the fabrication of sensors for zeptomolar concentrations and even for single molecule detection [ $\underline{3}, \underline{4}]$. Most recent sensing techniques utilize surface enhancement of plasmon excitations induced on metal nanostructures like Raman scattering, refractive index sensing, fluorescence- and infrared spectroscopy [5-11]. One of the most widely studied types of nanostructures is twodimensional gratings of metal nanoparticles arranged on top of a dielectric spacer layer and a supporting metal film [12-15]. This kind of nanostructure provides a very high grade of reproducibility and pronounced plasmon excitations that lead to ultra-high enhancement factors $[\underline{5}, \underline{16}]$. The combination of isolated metal nanoparticles with a mirroring metal film provides three advantages. First, the isolated particles are well understood in their relation between geometry and localized surface plasmon (LSP) frequency [17]. Second, the metallic mirror allows transportation of propagating surface plasmons (PSP), which can be coupled into the surface using a periodic grating of the nanoparticles. The third advantage is that the continuous metal film enhances the dipole-dipole interaction between the individual nanoparticles [12].

It has been shown that for small spectral differences between LSPs and PSP, the symmetric lineshapes change into asymmetric Fano-resonances. These Fano-resonances alter the line shape and effectively reduce the FWHM of the plasmon resonance, which can be used to enhance the sensitivity in refractive index sensing [15]. In case the distance between the LSP and the PSP resonance is approaching zero the resonances cancel each other out, called anticrossing [12]. The metallic mirror underneath the grating causes a red shift of the LSP resonance compared to particles directly deposited on a glass substrate [18]. According to recent literature, the interaction between plasmon resonances and their image excitation in a nearby metal film result in the phenomenon of plasmon hybridization [19-21]. Most commonly the hybridization of plasmon resonances is analyzed for multilayered nanocups or nanoshells and is characterized through the splitting of the plasmon modes into antibonding and bonding modes, respectively [22]. Since plasmon modes can be understood in terms of electromagnetic waves expanded into spherical harmonics, it is common to characterize them according to their different angular momenta, like dipole excitation, quadrupole excitation, and more [23]. Each of these excitations can interact with their image excitation when 
provided a metal film in near distance. The interaction splits each plasmon resonance in so called bonding and antibonding modes [24]. In the following we will demonstrate experimentally how mirror induced plasmon hybridization increases the complexity in the eigenmodes of plasmonic structures in the infrared and VIS regime.

\section{Material and Methods}

In this work, we experimentally observe plasmon hybridization in the spectral region between 0.1 and $1 \mathrm{eV}$ using infrared microscopy. Absorbance measurements are used to visualize the plasmon excitations. The structure presented here contains regular arrays of flat cylindrical gold nanoparticles with diameters ranging from 200 to $1500 \mathrm{~nm}$. The particles are connected to a $100 \mathrm{~nm}$ thick gold mirror via an insulating tantalum pentoxide nanoparticle of the same shape and size, located directly underneath the gold nanoparticle (see Fig. 1a). As reported previously, this arrangement of the spacer layer allows to perform electrochemical measurements in addition to a primary optical setup [14]. The size of the particles is chosen in order to tune the LSP resonance within the sensitive region of the infrared microscope, ranging from the near- to the mid-infrared region. Due to the cylindrical shape of the gold disks, the LSP resonances are independent from the polarization of the incident light. The structures were fabricated by sputtering four continuous layers on a glass substrate under argon atmosphere in the following order: $5 \mathrm{~nm}$ Ti adhesive layer, $100 \mathrm{~nm} \mathrm{Au,} 30 \mathrm{~nm} \mathrm{Ta} \mathrm{O}_{5}$ and $42 \mathrm{~nm} \mathrm{Au}$. The diameters of nanoparticles were defined using electron beam lithography, and formed in a top-down approach using reactive ion-beam etching. The electron beam resist deposits are removed afterwards through sonication in piranha solution $\left(3: 1 \mathrm{H}_{2} \mathrm{SO}_{4}: \mathrm{H}_{2} \mathrm{O}_{2}\right)$. IRabsorbance measurements performed with a dry-air-purged infrared microscope (Bruker Hyperion 3000) coupled to a FTIR spectrometer (Bruker Tensor 37). Using a liquid-nitrogen cooled mercury-cadmium telluride (MCT) detector, the spectra of each grating were calculated as the co-addition of 300 scans. A 64 x 64 focal plane array (FPA) detector was employed for spatially resolved measurements. Spectra were analyzed using the software package OPUS 7. 

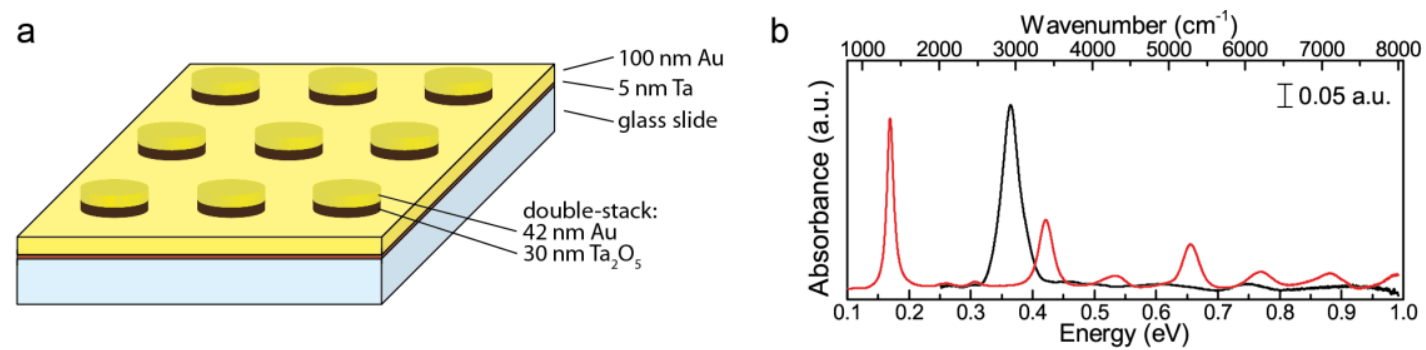

Fig. 1 a Scheme of two-dimensional arrays of $\mathrm{Ta}_{2} \mathrm{O}_{5} / \mathrm{Au}$ nanoparticles on top of a continuous gold layer. b Infrared absorption spectra of a structure on glass support (black) and of the same structure placed on a $100 \mathrm{~nm}$ thick gold layer (red), revealing the pronounced plasmon hybridization. The spectra were taken from double layered nanoparticle stacks with a diameter of $1500 \mathrm{~nm}$ having a grating constant of $2 \mu \mathrm{m}$.

In order to analyze the hybridization on the LSP modes, we compared the absorption of a nanoparticle grating placed on top of a glass slide without the continuous gold layer to the same grating placed on top of a $100 \mathrm{~nm}$ thick gold layer acting as mirror for plasmon excitations. An example of plasmon hybridization is shown in Fig.1b. The highest number of hybridized plasmon resonances was found for the largest particles (d 1500 nm), which exhibit the LSP resonance the energy of $0.18 \mathrm{eV}$, depicted in Fig.1b. While the substrate without gold film (black line) shows a pronounced dipole resonance at the energy of $0.36 \mathrm{eV}$, the according antibonding mode shows a strong red shift and is located at $0.18 \mathrm{eV}$. The dimensions of the nanoparticles were chosen for the present study because the resulting plasmon resonances are excited in the observable energy window $(0.1-1 \mathrm{eV})$ of the infrared microscope. LSP resonances of higher energies also provide the same amount of hybridized plasmon resonances, but the microscope cut off all resonances with energies higher than $1 \mathrm{eV}$.

In the second part, we investigated the infrared absorption signal of gratings that contain two differently sized nanoparticles. Smaller and bigger particles were mixed within a single grating, see Fig. 2. The structures were fabricated according to the protocol mentioned above. In order to analyze the interaction between two different LSP resonances, the diameter of one size is varied from $200-1500 \mathrm{~nm}$ while the second diameter had a constant value of $475 \mathrm{~nm}$. The crossing behavior of the two LSP peaks was tested on a regular grating with a constant of $2 \mu \mathrm{m}$, in arrays with a total size of $150 \times 150 \mu \mathrm{m}$. The absorbance measurements show that the two individual LSP modes can overlap freely and therefore they can be brought into any desired configuration of LSP resonances. This finding indicates that interaction between different LSP resonances within a grating is weaker than the interaction between LSP and PSP, where strong interaction leads to anticrossing of the resonances. 
In order to demonstrate that our structures are not only optically active in the infrared but also in the visible region, we arranged three arrays with different grating constants $G$ and nanoparticle diameters d. IR absorption measurements were performed for each grating using the MCT and FPA detector in order to check the LSP resonances and the spatial quality of the grating. Additionally, we took photographs of the gratings from different perspectives using a Canon EOS-D60 camera with and an EF-S60mm f/2.8 Macro USM lens. The change in color of the grating demonstrates that multiple modes of PSP and LSP resonances can be found within these gratings for the visible regime.

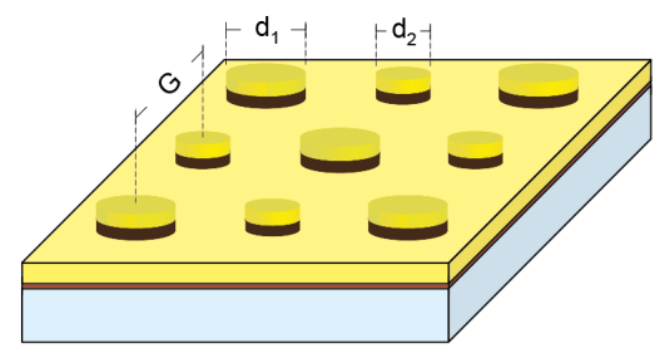

Fig. 2 Scheme of two-dimensional arrays of differently sized $\mathrm{Ta}_{2} \mathrm{O}_{5} / \mathrm{Au}$ nanoparticles on top of a continuous gold layer. $\mathrm{G}$ denotes the grating constant of the array i.e. distance between the nanoparticles, and $\mathrm{d}$ indicates the diameters of the discs.

\section{Results and Discussion}

The plasmon modes of nanoparticles interacting with a metal surface hybridize to form composite plasmon modes in a manner analogous to how atomic orbitals interact and form molecular orbitals [20]. The LSP resonance of the double layered nanoparticles with a diameter of $1500 \mathrm{~nm}$ placed on glass is located at $0.36 \mathrm{eV}$, as shown in the infrared absorption spectra in Fig 1b. A $100 \mathrm{~nm}$ thick gold film positioned below same-sized particles leads to a red-shift of the LSP resonance to $0.18 \mathrm{eV}$. Moreover, multiple resonances exhibiting higher energies are induced through the introduction of the gold film. In agreement with the theory, the plasmon resonances at $0.18 \mathrm{eV}$ and $0.42 \mathrm{eV}$ result from the hybridization of the dipole plasmon moment of the undisturbed plasmon resonance, which is located at $0.36 \mathrm{eV}[\underline{20}, \underline{21}]$. Whereas the quadrupole moment was not within the detection limit of the infrared microscope, the antibonding mode of the hybridized plasmon is assumed to be responsible for the third order plasmon resonance, located at $0.66 \mathrm{eV}$. The FWHM of the LSP mode with the lowest energy becomes lower through the hybridization, starting from $0.03 \mathrm{eV}$ on glass to 0.01 on the metal surface. Positions and FWHM values of three hybridized plasmon modes 
are collected in Fig. 3. For fitting of the resonances, a Cauchy-Lorentz distribution was employed according to the description for surface plasmon excitations using damped harmonic oscillators [25]. The limited energy window of the microscope only allowed the observation of three resonances for diameters greater than $950 \mathrm{~nm}$ with grating constants of 2 $\mu \mathrm{m}$. The smallest diameters up to $525 \mathrm{~nm}$ lead to the appearance of a single resonance. These plasmon resonances are direct observations of the self-sustained deformations of the electron liquid [20]. Because the liquid is incompressible, the only result of such deformations is the appearance on the surface of the nanoparticle [19]. The FWHM of the LSP modes and their respective distances contain both image-like contributions and dynamic effects arising from hybridization of the LSP from the flat cylindrical nanoparticles. First and second order resonances represent the antibonding and bonding modes of the dipole interactions, while the third order resonances represents the antibonding of the quadrupole excitation, respectively [24].

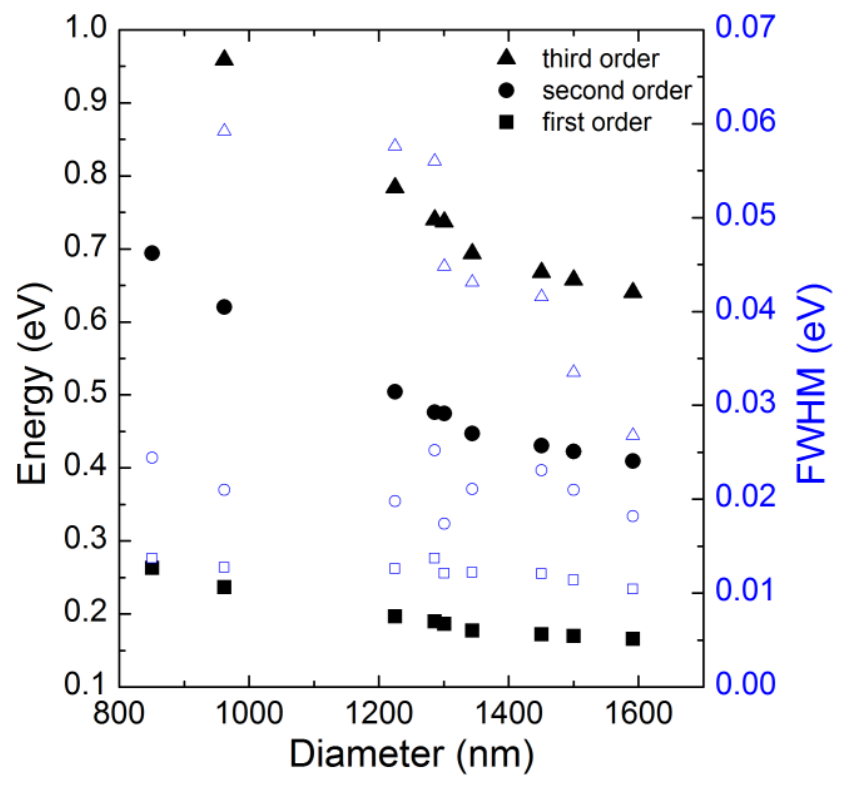

Fig. 3 Positions (black, left) and FWHM values (blue, right) of three hybridized plasmon modes for different nanoparticle diameters in gratings with a grating constant of $2 \mu \mathrm{m}$. The three orders arise through the hybridization of the dipole and the quadrupole plasmon exctitation. 
To analyze the interaction between individual LSP modes of differently sized nanoparticles within a single grating, the IR absorption spectra of single sized gratings are compared to structures containing mixtures of sizes, as demonstrated in Fig. 4. Resonance positions of LSP modes in the mixed grating do not change significantly from the single sized grating. This finding is a good proof that the lithographic production is feasible for producing nanoparticles with the same particle diameter for mixed and single sized gratings. The mixture in Fig. 4 shows the absorption of gratings containing particle diameters of $290 \mathrm{~nm}$ and $475 \mathrm{~nm}$, respectively. The drop in intensity and area of the plasmon resonances between gratings with larger diameters (green) to smaller diameters (blue) is attributed to the drop in surface coverage of the nanoparticles for each size $[\underline{26}, \underline{27}]$. For mixed-size arrays (red), the amount of particles of each size is only one half compared to the same-sized structures resulting in a drop in intensity of their LSP. Besides this intensity drop of the plasmon resonances, their line shape is well preserved in the IR spectra. The LSP mode in the single sized gratings with the smaller particles $(\mathrm{d}=290 \mathrm{~nm})$ is located at $0.61 \mathrm{eV}$ with a FWHM of $0.03 \mathrm{eV}$. In the mixed grating, these resonances are located at the same position with the same FWHM, whereas the maximum intensity drops from 0.16 a.u. to 0.076 a.u., corresponding to the fact that the number of nanoparticles with this size is only half in the mixture. There is a tendency that the FWHM is larger for plasmon resonances exhibiting higher energies, see Fig 3. Since these high energy plasmon resonances are excited by smaller nanoparticles, the increased FWHM can be explained by a higher relative error of the lithography procedure for smaller structures.
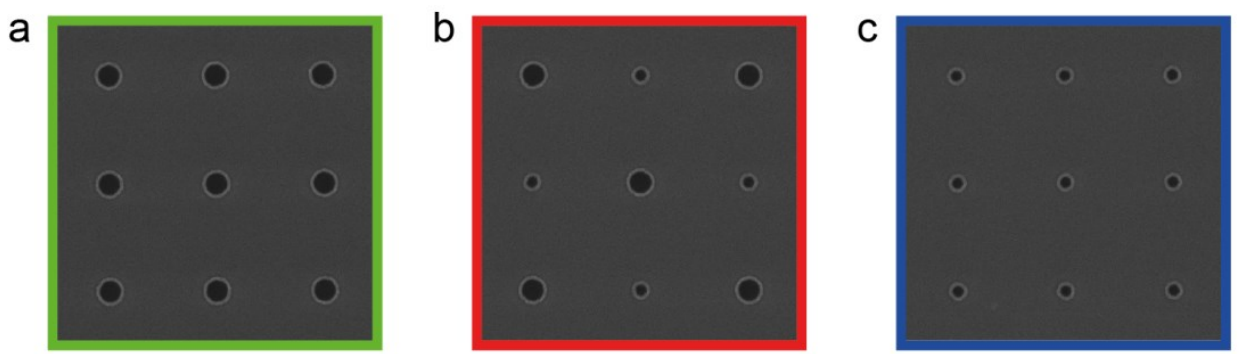

d

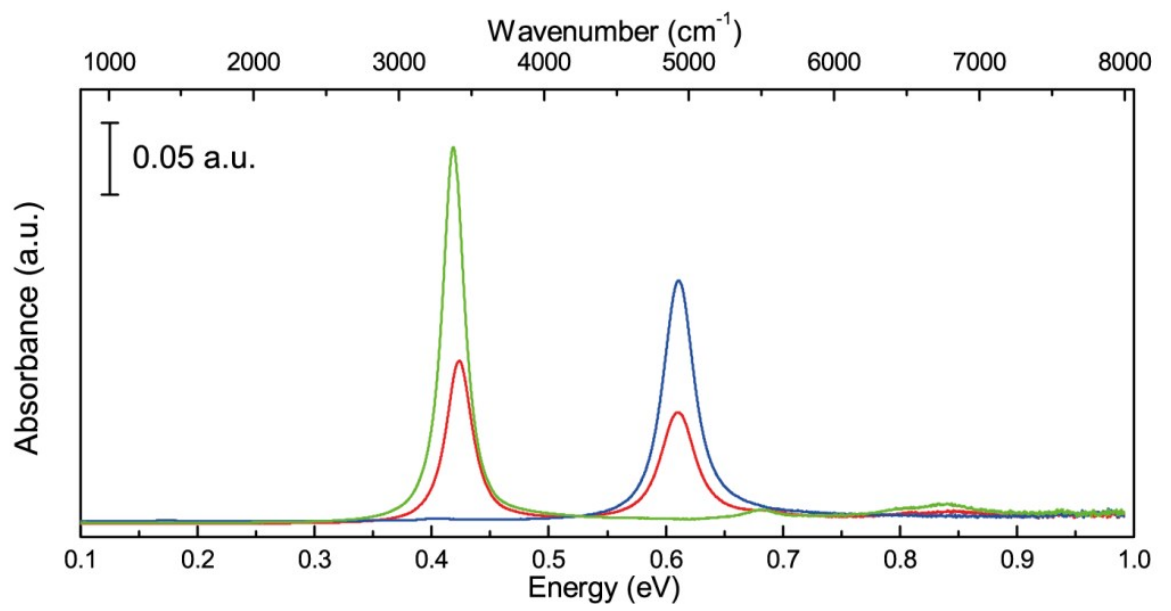


Fig. 4 SEM images of the two-dimensional array with disc diameters of a $475 \mathrm{~nm}, \mathbf{b} 475 \mathrm{~nm}$ and $290 \mathrm{~nm}$ as well as c $290 \mathrm{~nm}$. d IR absorption spectra of the gratings with same sized and differently sized nanoparticle stacks.

A systematic variation of the nanoparticle-diameters in the mixed-size gratings allowed studying the interaction between the respective LSP resonances. The diameter of every other nanoparticle $\left(d_{1}\right)$ is kept constant at a value of $475 \mathrm{~nm}$, while the diameter of the remaining discs $\left(\mathrm{d}_{2}\right)$ is varied in the steps of $200 \mathrm{~nm}, 290 \mathrm{~nm}, 350 \mathrm{~nm}, 400 \mathrm{~nm}, 475 \mathrm{~nm}, 525 \mathrm{~nm} 850 \mathrm{~nm}$, $1225 \mathrm{~nm}$ and $1500 \mathrm{~nm}$. In Fig. 5, the IR absorbance spectra of the different mixtures are plotted, starting with the smallest particles on top and the largest particles at the bottom of the figure. Whereas the smaller particles only show one LSP resonance, the mixture containing particles with a diameter of $850 \mathrm{~nm}$ exhibits three resonances, two of which are attributed to the individual response of the $850 \mathrm{~nm}$ particles. The mixtures containing the two largest particles reveal four pronounced plasmon excitations, from which three are attributed to the particle with the larger diameter in the mixture. The results show that neither the first order nor the second order of the LSP resonances resulting from varied diameter $\mathrm{d}_{2}$ exhibits any interaction with the resonance from the particle with constant diameter $\mathrm{d}_{1}$, since the shape of the resonances is not affected by the mixture and the resonances can overlap freely. Therefore 


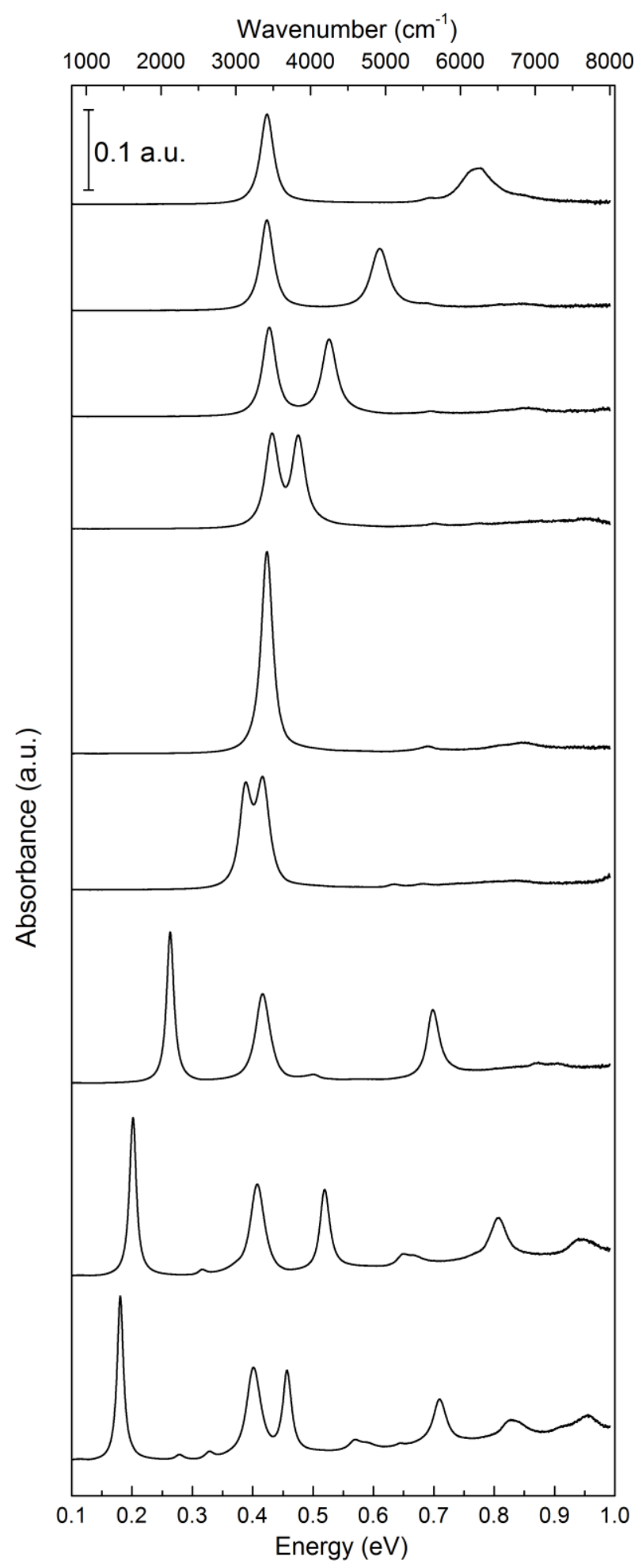

Fig.5 IR absorption spectra of gratings with differently sized nanoparticles on top of a continuous gold layer as depicted in Fig. 2. While $d_{1}$ was kept constant at $475 \mathrm{~nm}, \mathrm{~d}_{2}$ was increased from $200 \mathrm{~nm}$ (top) to $1500 \mathrm{~nm}$ (bottom). See text for details. 
such gratings can be used to create any desired configuration of LSP resonances. In contrast to the earlier described interaction between LSP and PSP [12], the interaction between two individual LSP modes allows to combine resonances which are located in close distance to each other. Furthermore, gratings of rather large particles can potentially benefit from this autonomous behavior of the resonance, when including small particles in the gap between the larger particles.

a
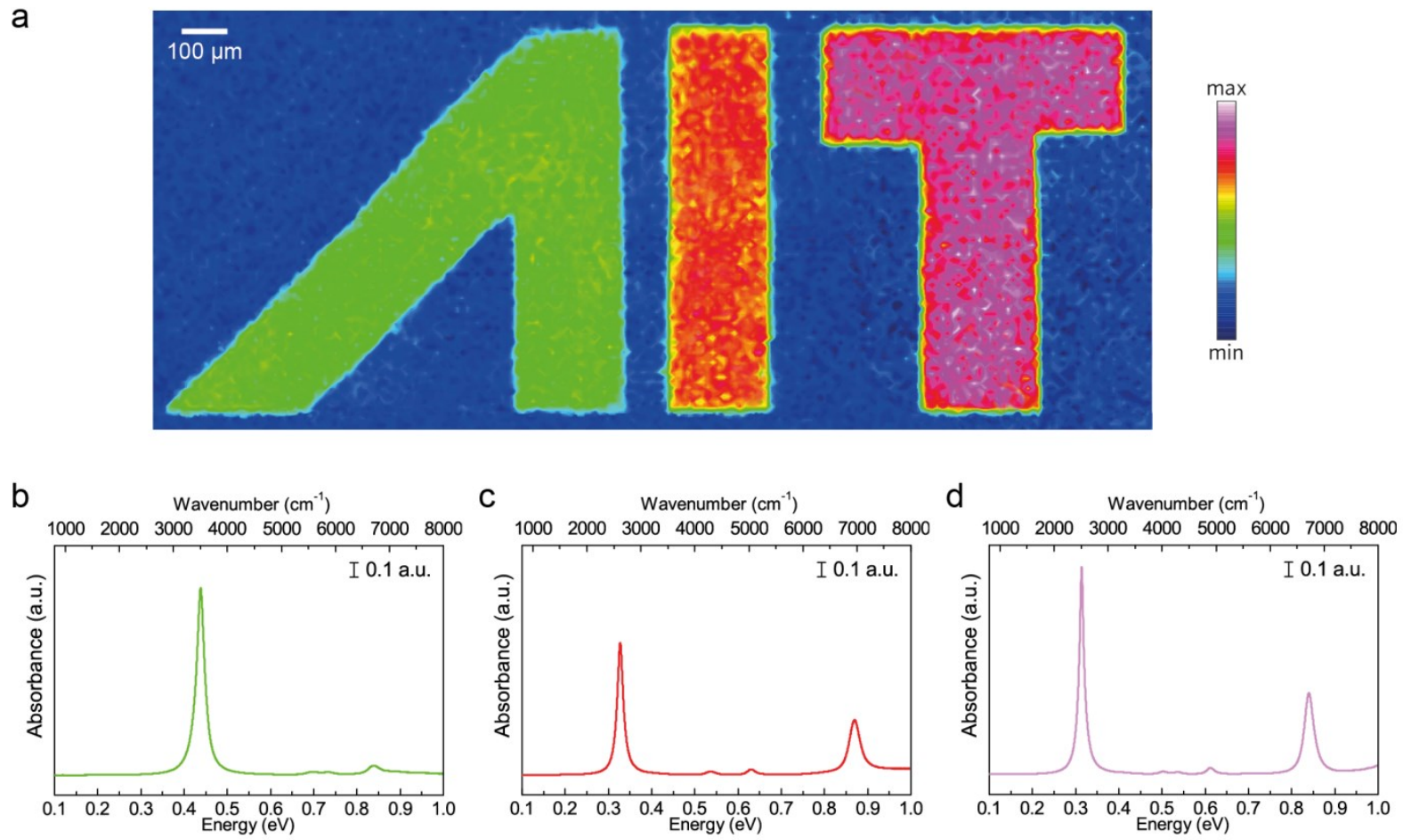

Fig. 6 a Two-dimensional map acquired with a FPA detector depicting the total IR intensity of nanostructures with grating constants $\mathrm{G}$ : $A=1200 \mathrm{~nm}, \mathrm{I}=800 \mathrm{~nm}, \mathrm{~T}=1000 \mathrm{~nm}$; diameters $\mathrm{d}$ : $\mathrm{A}=650 \mathrm{~nm}, \mathrm{I}=450 \mathrm{~nm}, \mathrm{~T}=650 \mathrm{~nm}$. The observed area of $2.1 \times 0.9 \mathrm{~mm}$ features nanostructured arrays with different particle diameters and grating constants. IR spectra of nanostructures with b grating constant $\mathrm{G}=1200 \mathrm{~nm}$ and particle diameter $\mathrm{d}=650 \mathrm{~nm}, \mathbf{c} \mathrm{G}=800 \mathrm{~nm}$ and $\mathrm{d}=450$ as well as $\mathbf{d} \mathrm{G}=1000$ and $\mathrm{d}=650 \mathrm{~nm}$, representing the nanostructured arrays indicated in $\mathbf{a}$, respectively.

In order to test the production quality of larger arrays in the mm scale, FPA infrared absorption measurements were performed with a spatial resolution of $2.7 \mu \mathrm{m}$. For this purpose, three different combinations of grating periods and particles sizes were fabricated on the same surface. To ensure facile discrimination of the separate gratings, their overall shape was chosen in the form of three different letters, which are A, I and T. The grating constants and particle diameter combinations of the individual structures are $A(G=1200 \mathrm{~nm}, \mathrm{~d}=650$ $\mathrm{nm}), \mathrm{I}(\mathrm{G}=800 \mathrm{~nm}, \mathrm{~d}=450 \mathrm{~nm})$ and $\mathrm{T}(\mathrm{G}=1000 \mathrm{~nm}, \mathrm{~d}=650 \mathrm{~nm})$. The IR absorbance spectra of 
the individual gratings are shown in Fig 6 b-d. It is clearly visible that different grating constants strongly influence the relation between diameter and LSP resonance position. Fig. 6a shows a two-dimensional map acquired with a FPA detector, depicting the total IR intensity of the surface. The letter $\mathrm{T}$ exhibits the highest total intensity (magenta) with first and second order plamonic resonances and higher band intensities than the letter I (red). The letter A, with only a first order plasmonic excitation features the lowest total IR intensity (green).
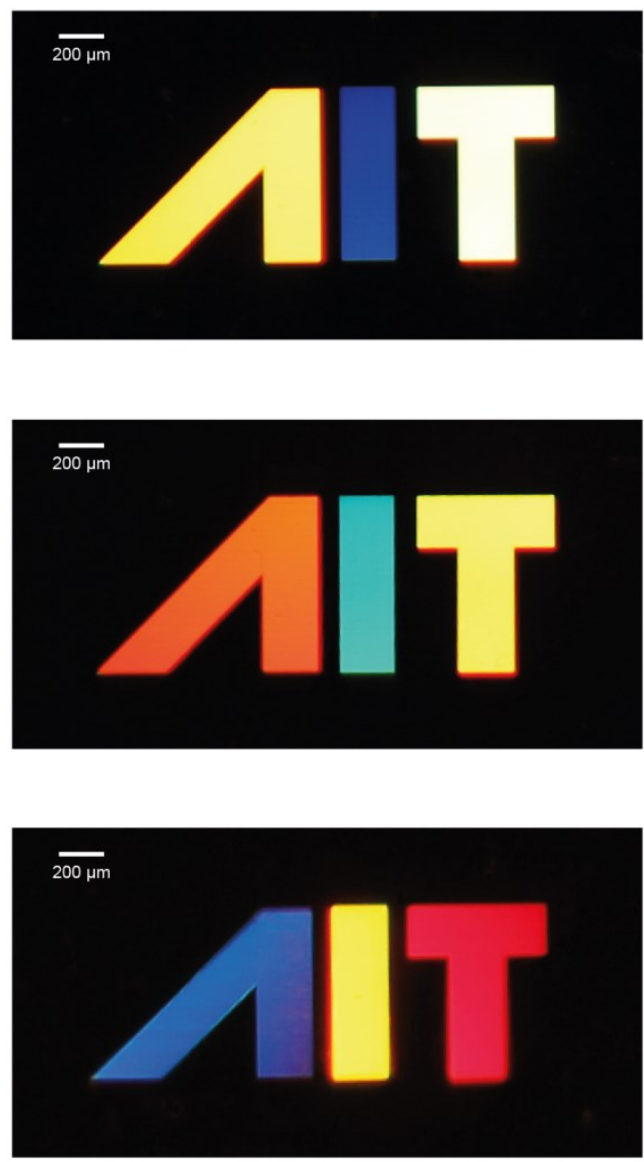

Fig. 7 Photographs of a nanostructured surface consisting of three letters with a total area of $2.8 \times 1.5 \mathrm{~mm}$. Each letter is made of a gold nanoparticle array on top of a flat gold surface (A: $\mathrm{G}=1200 \mathrm{~nm}, \mathrm{~d}=650 \mathrm{~nm} / \mathrm{I}: \mathrm{G}=800 \mathrm{~nm}, \mathrm{~d}=450 \mathrm{~nm} / \mathrm{T}: \mathrm{G}=1000 \mathrm{~nm}, \mathrm{~d}=650 \mathrm{~nm}$ ). The photographs are taken under normal incidence using a white light source. Each picture shows the structures illuminated from a different angle. The gold surface appears black, due to the near distance of the camera and the mirroring nature of the flat gold film.

In order to demonstrate the plasmonic activity in the visible region we took several photographs of the structure which was used for the FPA IR microscopy measurements. Whereas the FPA spectra provide information about the infrared absorption of the structure, the photos give insight into its plasmon resonances in the VIS regime. Fig. 7 shows 
photographs from three different perspectives of this structure, which was illuminated using a white light source. While the incident angle of the illumination is altered, all pictures are taken under angles in close proximity to normal incidence to the surface. Due to the near distance of the camera to the surface and the reflecting properties of the flat gold surface, the $100 \mathrm{~nm}$ thick gold film without gratings appears black in the pictures. Variations in the angle of the incident light result in color changes in the appearance of the nanoparticle gratings, which is attributed to multiple plasmonic modes in the VIS regime resulting from the interaction between LSP and PSP resonances. These plasmonic resonances in the VIS regime are predominantly governed by the grating constants, whereas the position of the plasmon excitations in the infrared is primarily determined by the stack diameter. The pictures visualize the complex optical properties of such nanoparticle gratings, and show their strong dependence on the angle of both the illumination and observation.

\section{Conclusions}

In conclusion, regular gratings of double layered nanoparticle stacks have been fabricated using a sputter machine, e-beam lithography and reactive ion etching. The approach provides the highest grade of reproducibility and offers easy control and uniformity in the size distribution of the particles. We observed plasmon hybridization by comparing nanostructures arranged on substrates providing a supporting gold film to substrates without a gold film. In accordance to theoretical predictions, the plasmon mode with the lowest energy is strongly red shifted, representing the antibonding mode of the interacting dipole excitations. Moreover, multiple plasmon excitations arise due to the interaction of the dipole and quadrupole plasmon excitations in the gold nanoparticles. Arrays containing single diameters as well as mixtures of diameters within a single array are studied using infrared microscopy. The resulting absorption spectra were discussed in terms of localized surface plasmon excitations. Alternating diameters of nanoparticles within a single array reveal that there is no interaction between the corresponding LSP modes, which allows autonomous tuning of each resonance. Due to plasmon hybridization and the interaction between LSP and PSP modes the structures show pronounced plasmon excitations in the infrared as well as in the visible regime. Therefore, the platform provides versatile options for designing sensors that combine different spectral regions. 


\section{Acknowledgements}

The authors thank Stephanie Alteneder for providing the utilized photo camera with an adequate lens system. Partial support for this work was provided by ZIT, Centre of Innovation and Technology of Vienna. The authors would also like to thank the City of Vienna (MA 7) for financial support of this work.

\section{References}

1. Anker JN, Hall WP, Lyandres O, Shah NC, Zhao J, Van Duyne RP (2008) Biosensing with plasmonic nanosensors. Nat Mater 7 (6):442-453.

2. Freestone I, Meeks N, Sax M, Higgitt C (2007) The Lycurgus Cup - A Roman nanotechnology. Gold Bull 40 (4):270-277.

3. Mayer KM, Hao F, Lee S, Nordlander P, Hafner JH (2010) A single molecule immunoassay by localized surface plasmon resonance. Nanotechnology 21 (25).

4. Adato R, Yanik AA, Amsden JJ, Kaplan DL, Omenetto FG, Hong MK, Erramilli S, Altug H (2009) Ultrasensitive vibrational spectroscopy of protein monolayers with plasmonic nanoantenna arrays. Proc Natl Acad Sci 106 (46):19227-19232.

5. Adato R, Altug H (2013) In-situ ultra-sensitive infrared absorption spectroscopy of biomolecule interactions in real time with plasmonic nanoantennas. Nat Commun 4.

6. Bauch M, Dostalek J (2013) Collective localized surface plasmons for high performance fluorescence biosensing. Opt Express 21 (17):20470-20483.

7. Chu Y, Banaee MG, Crozier KB (2010) Double-Resonance Plasmon Substrates for Surface-Enhanced Raman Scattering with Enhancement at Excitation and Stokes Frequencies. ACS Nano 4 (5):2804-

2810.

8. D’Andrea C, Bochterle J, Toma A, Huck C, Neubrech F, Messina E, Fazio B, Maragò OM, Di Fabrizio

E, Lamy de La Chapelle M, Gucciardi PG, Pucci A (2013) Optical Nanoantennas for Multiband SurfaceEnhanced Infrared and Raman Spectroscopy. ACS Nano:3522-3531.

9. Nedelkovski V, Schwaighofer A, Wraight CA, Nowak C, Naumann RLC (2013) Surface-Enhanced Infrared Absorption Spectroscopy (SEIRAS) of Light-Activated Photosynthetic Reaction Centers from Rhodobacter sphaeroides Reconstituted in a Biomimetic Membrane System. J Phys Chem C 117 (32):16357-16363.

10. Offermans $P$, Schaafsma MC, Rodriguez SRK, Zhang Y, Crego-Calama M, Brongersma SH, Gómez Rivas J (2011) Universal Scaling of the Figure of Merit of Plasmonic Sensors. ACS Nano 5 (6):51515157.

11. Zou C, Larisika M, Nagy G, Srajer J, Oostenbrink C, Chen X, Knoll W, Liedberg B, Nowak C (2013) Two-Dimensional Heterospectral Correlation Analysis of the Redox-Induced Conformational Transition in Cytochrome c Using Surface-Enhanced Raman and Infrared Absorption Spectroscopies on a Two-Layer Gold Surface. J Phys Chem B 117 (33):9606-9614.

12. Chu Y, Crozier KB (2009) Experimental study of the interaction between localized and propagating surface plasmons. Opt Lett 34 (3):244-246.

13. Félidj N, Aubard J, Lévi G, Krenn JR, Schider G, Leitner A, Aussenegg FR (2002) Enhanced substrate-induced coupling in two-dimensional gold nanoparticle arrays. Phys Rev B 66 (24):245407. 14. Frank P, Srajer J, Schwaighofer A, Kibrom A, Nowak C (2012) Double-layered nanoparticle stacks for spectro-electrochemical applications. Opt Lett 37 (17):3603-3605.

15. Lodewijks K, Ryken J, Roy W, Borghs G, Lagae L, Dorpe P (2013) Tuning the Fano Resonance Between Localized and Propagating Surface Plasmon Resonances for Refractive Index Sensing Applications. Plasmonics 8 (3):1379-1385. 
16. Srajer J, Schwaighofer A, Ramer G, Rotter S, Guenay B, Kriegner A, Knoll W, Lendl B, Nowak C (2013) Double-layered nanoparticle stacks for surface enhanced infrared absorption spectroscopy. Nanoscale:DOI: 10.1039/C1033NR04726A.

17. Oskooi AF, Roundy D, Ibanescu M, Bermel P, Joannopoulos JD, Johnson SG (2010) Meep: A flexible free-software package for electromagnetic simulations by the FDTD method. Comput Phys Commun 181 (3):687-702.

18. Holland WR, Hall DG (1984) Frequency Shifts of an Electric-Dipole Resonance near a Conducting Surface. Phys Rev Lett 52 (12):1041-1044.

19. Nordlander P, Oubre C, Prodan E, Li K, Stockman MI (2004) Plasmon Hybridization in Nanoparticle Dimers. Nano Lett 4 (5):899-903.

20. Nordlander P, Prodan E (2004) Plasmon Hybridization in Nanoparticles near Metallic Surfaces. Nano Lett 4 (11):2209-2213.

21. Prodan E, Radloff C, Halas NJ, Nordlander P (2003) A Hybridization Model for the Plasmon Response of Complex Nanostructures. Science 302 (5644):419-422.

22. Wollet L, Frank B, Schaferling M, Mesch M, Hein S, Giessen H (2012) Plasmon hybridization in stacked metallic nanocups. Opt Mater Express 2 (10):1384-1390.

23. Myroshnychenko V, Rodriguez-Fernandez J, Pastoriza-Santos I, Funston AM, Novo C, Mulvaney P, Liz-Marzan LM, Garcia de Abajo FJ (2008) Modelling the optical response of gold nanoparticles. Chem Soc Rev 37 (9):1792-1805.

24. Huang J-S, Kern J, Geisler P, Weinmann P, Kamp M, Forchel A, Biagioni P, Hecht B (2010) Mode Imaging and Selection in Strongly Coupled Nanoantennas. Nano Lett 10 (6):2105-2110.

25. Bosman M, Ye E, Tan SF, Nijhuis CA, Yang JKW, Marty R, Mlayah A, Arbouet A, Girard C, Han M-Y (2013) Surface Plasmon Damping Quantified with an Electron Nanoprobe. Sci Rep 3.

26. Maier T, Brückl H (2009) Wavelength-tunable microbolometers with metamaterial absorbers. Opt Lett 34 (19):3012-3014.

27. Maier T, Brueckl H (2010) Multispectral microbolometers for the midinfrared. Opt Lett 35 (22):3766-3768. 